\title{
Obtaining new substituted 4-dimethylamino-2-(naphth-1-yl)-phenyl- 1-(2-chloroquinolin-3-yl)-butan-2-ol having activity against resistant strains of $M$. Tuberculosis
}

\author{
(C) Anton V. Omelkov, ${ }^{+}$Evsey A. Ruchko, and Vladimir E. Fedorov \\ JSC "Pharm-Synthesis". Enthusiasts Road, 38. Moscow, 105118. Russia. \\ E-mail: omelkov@mail.ru,Vfedorov@pharm-sintez.ru
}

\begin{abstract}
* Supervising author; ${ }^{+}$Corresponding author
Keywords: lithium diethylamide, diarylquinolines, 4-dimethylamino-2-(naphth-1-yl)-phenyl-1-(2chloroquinolin-3-yl)-butan-2-ol, 3-benzyl-2-chloroquinolines, 3-(dimethylamino)-1-(1-naphthyl)1-propanone, tuberculosis.
\end{abstract}

\begin{abstract}
The discovery in 2002 among derivatives of 6-bromo-3-benzyl-2-methoxyquinolines the highly active compounds against $M$. Tuberculosis bacterium and its drug-resistant strains led to the creation of the innovative anti-TB drug TMS-207 in 2013 by Janssen-Cilag, Bedaqueline ${ }^{\circledR}(1 R, 2 S)^{*}$-1-(6-bromo-2methoxy-3-quinolyl)-4-dimethylamino-2-(naphth-1-yl)-1-phenyl-butane-2-ol. Developing this area of research in our laboratory we set ourselves the task of further modifying the structure of Bedakvelin ${ }^{\circledR}$ in order to obtain new not described patent-capable compounds with greater bioavailability and fewer side effects. To achieve this goal, it was planned to synthesize a number of derivatives of TMS-207 with a change in the substituents in the quinoline ring. Among the structural analogues of Bedakvelin ${ }^{\circledR}$ described in the literature, derivatives of 2-chloroquinolines were absent. The synthesis and study of properties derived from this particular structure has become the goal of this study. It was decided to preserve as the active molecule fragment of the base part the Bedakvelin ${ }^{\circledR}$ which was optimal for derivatives of 2-methoxy-quinolinolin order. The scheme of obtaining the target compounds that we have chosen is practically uncontested if we take into account the boundary conditions for the cost of raw materials and minimize the number of synthesis stages. The resulting diastereomeric pairs differ in activity in M. Tuberculosis. RSSR diastereomeric pairs had greater activity against mycobacteria than RRSS diastereomeric pairs. Stereoselective or stereospecific preparation of such compounds with a good yield is desirable when producing the necessary tertiary alcohols.

The aim of this work was to determine all the reaction conditions that affect the yield of the target 4-dimethylamino-2-(naphth-1-yl)-phenyl-1-(2-chloroquinolin-3-yl)-butan-2-ols, and the determination of the possibility of a stereospecific or stereoselective method for the synthesis of such compounds.
\end{abstract}

\section{References}

[1] V. Baptiste, C. Crauste, M. Flipo et al. Tuberculosis: The drug development pipeline at a glance. Eur. J. Med. Chem. 2012. Vol.51. P.1-16.

[2] A.V. Omelkov, V.F. Fedorov. The Effect of Lithium, Cerium (III), Copper (I) Chlorides on the Synthesis of 1-(2-Chloro-3-quinolyl)-4-dimethylamino-2-(1-naphtyl)-1-phenyl-butan-2-ols. Book of abstracts, " $2^{\text {nd }}$ Russian Conference on Medicinal Chemistry". MedChem-2015, Novosibirsk. 2015. P.240.

[3] B.J. Wakefield. Organolithium Methods. Academic Press. 1988.

[4] W.E. Parham, R.M. Piccirilli. Selective halogen-lithium exchange in 2,5-dibromobenzenes and 2,5dibromopyridine. Journal of Organic Chemistry. 1977. Vol.42. No.2. P.257-260.

[5] E.-C. Koch. Acid-Base Interactions in Energetic Materials: I. The Hard and Soft Acids and Bases (HSAB) Principle "Insights to Reactivity and Sensitivity of Energetic Materials. Propellants, Explosives, Pyrotechnics. 2005. Vol.30. No.1. P.5-16.

[6] R.G. Pearson. Hard and soft acids and bases, HSAB, part 1: Fundamental principles. Journal of Chemical Education. 1968. Vol.45. No.9. P.581. 